\title{
Primary Extra-Nodal Non-Hodgkin Lymphoma of the Cheek: A Rare Case Report and Literature Review
}

\author{
F. E. Rizkou, D. Berrada El Azizi, S. Salhi, O. Benhoummad, Y. Rochdi, A. Raji
}

\section{ABSTRACT}

Non-Hodgkin's lymphomas are a group of highly diverse lymphoproliferative malignancies and have great tendency to involve in organs and tissues that typically don't contain lymphoid cells. Primary extra-nodal NHL of the oral cavity are thin on the ground, and cheek location is one of the rarest intraoral sites involved. The majority of oral NHLs reported are of diffuse large B-cell type. The present case highlights one of the scarce forms of primary extra-nodal non-Hodgkin; cheek location reveled by a swelling in the right cheek. Routine laboratory tests were within normal limits and clinical examination showcases painless, poorly limited mass. CT and MRI were performed revealing an enhanced tissue lesion with muscular infiltration and no bone lysis. An initial biopsy of the lesion was determined insignificant. A resection biopsy was evaluated using histologic techniques and immunohistochemistry, which established the proper diagnosis of extra-nodal diffuse large B-cell lymphoma. In view of their malignant nature and the fact that only histopathological examination can define the diagnosis, we report this case to aid an early recognition, diagnosis, and treatment, that are essential for a patient's survival.

Keywords: Cheek, extra-nodol, lymphoma, non-hodgkin.
Submitted : December 24, 2021

Published : February 23, 2022

ISSN: $2593-8339$

DOI: $10.24018 /$ ejmed.2022.4.1.1177

\section{F.E. Rizkou *}

ENT-Neck and Head Surgery Department, University Medical Center Mohammed VI, Marrakech, Morocco.

(e-mail: fati94rizkou@gmail.com)

D. Berrada El Azizi

ENT-Neck and Head Surgery Department, University Medical Center Mohammed VI, Marrakech, Morocco. (e-mail:

douniaberradaelazizi@gmail.com)

S. Salhi

ENT-Neck and Head Surgery Department, University Medical Center Mohammed VI, Marrakech, Morocco.

(e-mail: salma.salhi@edu.uca.ac.ma)

O. Benhoummad

ENT-Neck and Head Surgery

Department, University Hospital of

Agadir, Faculty of Medicine and

Pharmacy of Agadir, Morocco.

(e-mail: benhoummadorl@gmail.com)

Y. Rochdi

ENT-Neck and Head Surgery Department, University Medical Center Mohammed VI, Marrakech, Morocco.

(e-mail: rochdi_86@hotmail.com)

A. Raji

ENT-Neck and Head Surgery

Department, University Medical Center Mohammed VI, Marrakech, Morocco.

(e-mail: raji.abdelaziz@gmail.com)

*Corresponding Author

\section{INTRODUCTION}

Lymphomas are a group of highly various malignant neoplasms of lymphatic system, traditionally divided into two subtypes, Hodgkin lymphomas (HL) and non-Hodgkin lymphomas (NHL). The second one has great tendency to affect organs and tissues that do not ordinarily contain lymphoid cells, describing primary extra-nodal diseases. The last-mentioned fills up to $10 \%$ to $20 \%$ of all NHLs of the head and neck region, with the cheek location being highly unorthodox [1]-[4].

The variety and the scarcity of the clinical entity in the oral region, with no pathognomonic features often leads to misdiagnosis or a late one, delaying the adequate treatment for the patient which directly impairs the prognosis [4].

This study reports a case of a patient with primary extranodal diffused large $\mathrm{B}$ cell non-Hodgkin lymphoma in the cheek.

\section{CASE REPORT}

72 years old female patient with history of endometrial cancer; in which she was treated with radio/chemotherapy about 14 years ago; was admitted to the ENT department for the management of a slowly growing swelling over her right cheek that dates back to 6 months prior, associated with 
intermittent pain and headache. During this period the patient also accused weight loss, fever and weakness. The clinical examination revealed a firm, poorly circumscribed, painless mass on the right cheek reaching up to right lower jaw without limitation of the opening of mouth nor peripheral facial palsy "Fig. 1". The overlying skin was tense but normal along with the Stenon's duct. There was no lymph node enlargement. Her Lab test showed a VES of $76 \mathrm{~mm} 1^{\circ}$-hour, C-reactive protein of $1,51 \mathrm{mg} / \mathrm{dl}$ and LDH of 238 . Complete blood count noted no abnormalities with a count of white blood cells of $7,62 \times 103 / \mathrm{microL}$ ), neutrophils $72 \%$ and lymphocytes of $19.7 \%$.

CT scan showcased an $11 \mathrm{~mm}$ poorly limited thickening of tissue density in the cheek area, enhanced after injection of contrast product. This thickening comes with intimate contact with the maxillary bone without bone destruction without any laterocervical node involvement and metastasis "Fig.2".

MRI imaging revealed a hypointense T1 and T2 and hyperintense diffusion tissue lesion of the right cheek of $4 \mathrm{~cm}$, moderately enhanced by contrast, with muscular infiltration and no notable bone lysis "Fig.3".

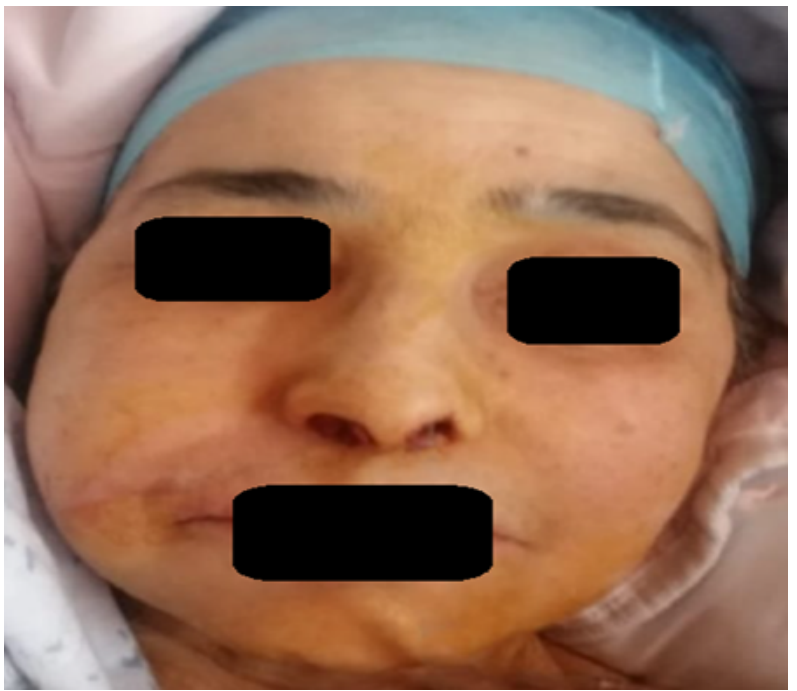

Fig. 1. Clinical aspect of the cheek mass.

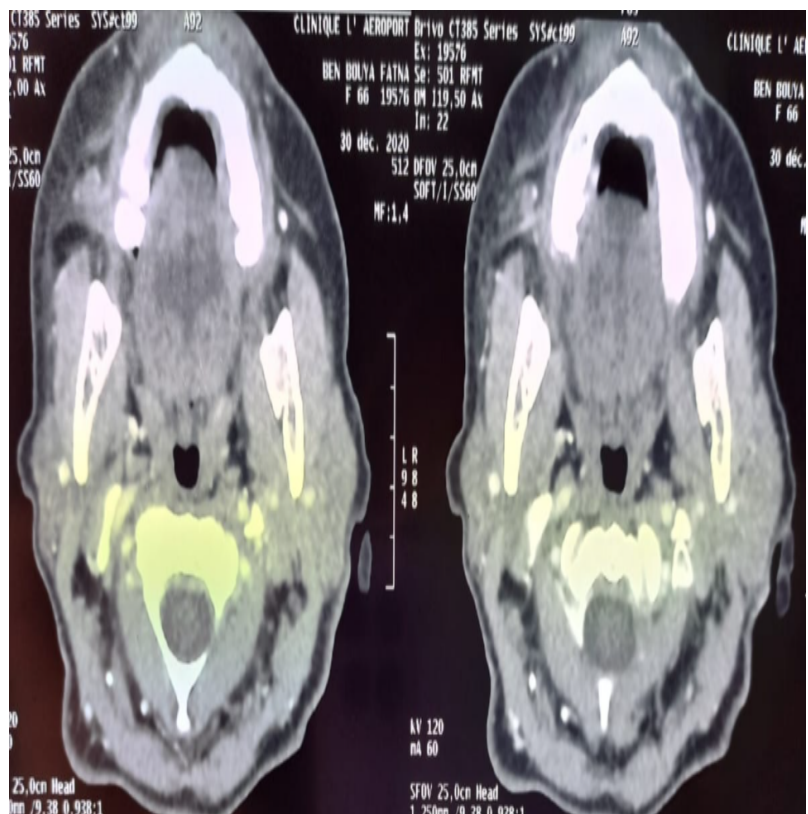

Fig. 2. Axial CT scan of a poorly limited tissue density cheek mass.
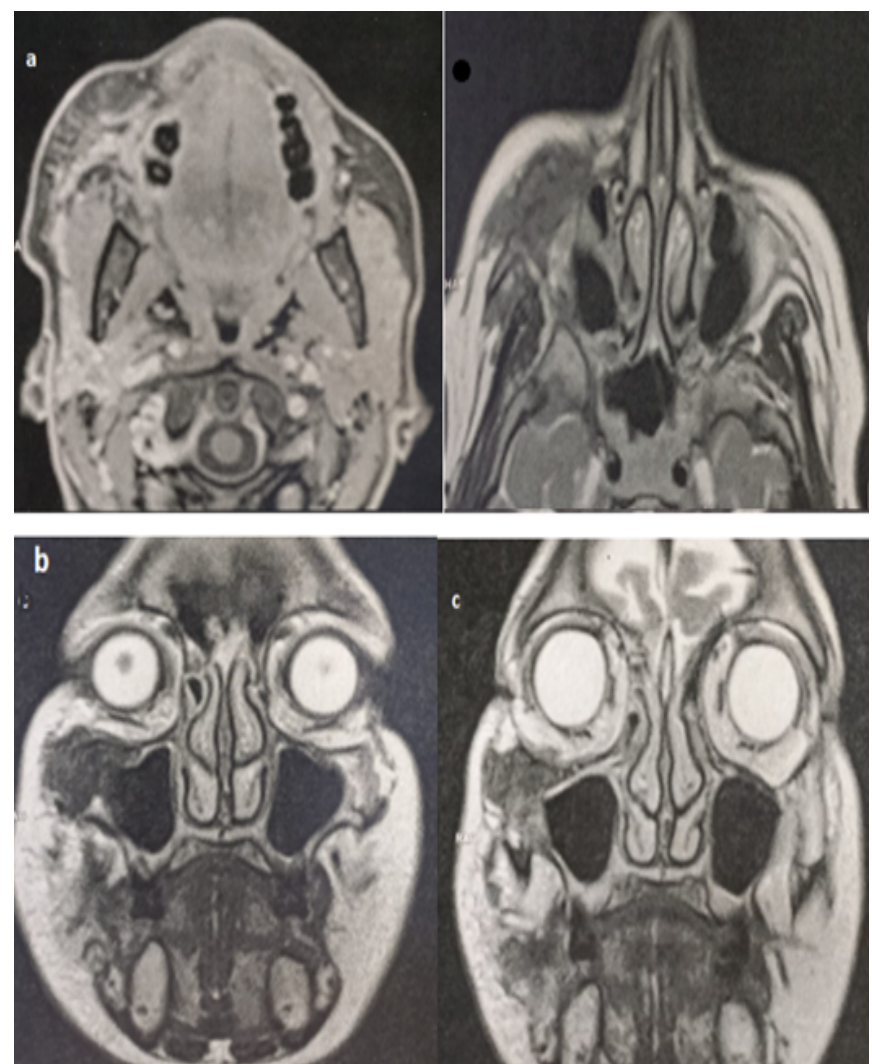

Fig. 3. MRI imaging: (a) axial T1 showcasing an hypointense cheek mass, (b) coronal T1 revealing as well an hypointense mass, (c) coronal T2 showing an hypointense cheek mass.

The patient underwent a local biopsy without no proper diagnosis which lead to a general anesthesia resection. An upper vestibular incision was performed to locate the tumor that appears brownish and ovoid. To note that the follow-up was unremarkable.

The histopathological typing revealed a large diffuse B cell non-Hodgkin's lymphoma. The immunophenotyping showed B-cell type CD20, CD30 and Ki67 positive and CD3 and CD138 negative "Fig.4".
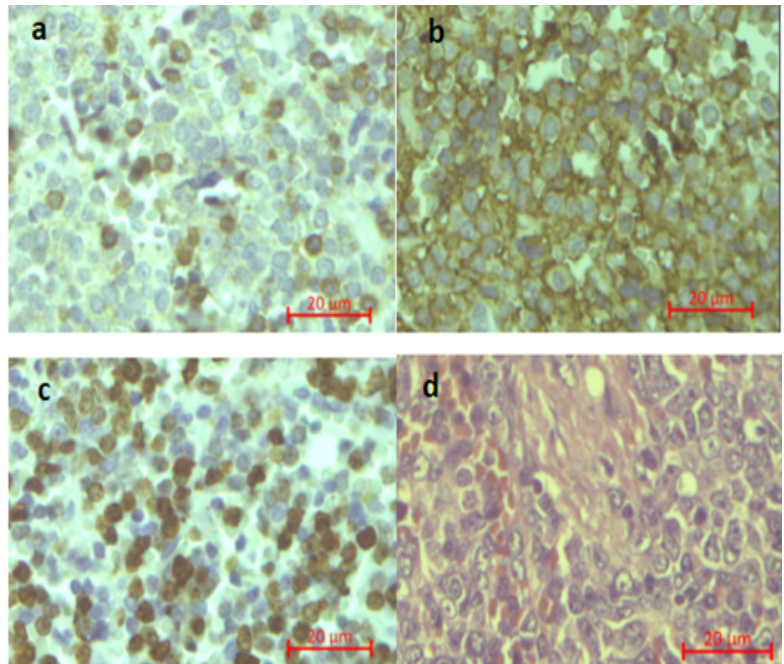

Fig.4. immunophenotyping: (a) anti CD3 antibody (lymphocytes T marker), (b) anti CD20 antibody (lymphocytes B marker), (c) anti Ki67 (proliferation index), (d) hemalum eosin coloration.

With the diagnosis in hand, the patient underwent a complete workup to rule out other systemic lesions, thereby supporting the primary nature of the lesion, and received RCHOP chemotherapy protocol. 


\section{DiscuSSION}

In terms of head and neck malignancies, lymphomas represent the third most common group of lesions, followed by squamous cell carcinoma and salivary gland neoplasm [1][3]. They are classically divided into two subtypes, Hodgkin lymphomas (HL) and non-Hodgkin lymphomas (NHL). The later subgroup that arises from lymphoreticular histogenesis and differentiation, may be developed in lymph nodes or other organs, either by spread from a lymphatic site or as a manifestation of primary extra-nodal disease [4], [5].

$10 \%$ to $20 \%$ of all NHLs of the head and neck region are primary extra-nodal (PE), predominantly in Waldeyer's ring, followed by salivary glands, particularly the parotid gland, the oral cavity location remains very uncommon. Among various intra-oral sites, the involvement of the cheek or the buccal mucosa has been reported quite infrequently [7]-[10].

The most common extra-nodal NHL's phenotype is the diffuse large B cell lymphoma (DLBCL) [7], [10].

These malignancies generally demonstrate a wide age range, occurring during adulthood and rarely in children. The sex ratio of extra-nodal lymphoma favors male preponderance [11], [12].

The development of NHLs is wildly discussed and contributed to various components, counting immunodeficiency, Epstein Barr virus, human T-cell leukemia virus 1 , Helicobacter pylori disease, hepatitis $\mathrm{C}$, deregulation of the cell cycle, as well as host vulnerability and susceptibility factors (congenital or acquired). [4], [13], [14].

Scarce maybe, with no pathognomonic clinical features of the cheek lymphoma, the prevalent clinical presentation is a local asymptomatic mass, though it can be associated with pain, fever and weight loss. Swelling, ulceration, hearing loss or earache were described and within its evolution, facial swelling and visual disturbance, destruction of bones and extension into alveolar bone, the paranasal sinuses, paranasopharyngeal space, hard palate, the orbit, infra-temporal fossa, and intracranial cavity were also described possibilities [4], [10], [17]-[19].

It has to be mentioned that NHL spreads most commonly to non-adjacent nodes with a common abdominal involvement and this multiorgan inclusion comes with a towering mortality rate, thus the poor clinical outcome [16], [20]-[23].

Histopathological examination of the specimen with immunohistochemistry is the key to authenticate the diagnosis and abolish the differentiation diagnosis. A deep biopsy is a choice, usually taken under local anesthesia, resection in the other hand is usually be performed under general one. That been said and to allow immunohistochemical staining, the biopsy specimen shouldn't be fixed and out to be proceed freshly. Albeit the usefulness of fine-needle aspiration for the orientation of the diagnosis especially in case of associated node, it may be the cause of both faulty positive and negative results. [4], [24][26].

In terms of paraclinical examination, blood work can identify anemia and quantitative changes in the leucocyte line, transaminases, creatinine, lactate dehydrogenase and b2microglobulin indicate or at least orient to the involvement of the respective organs. Increments in calcium levels and alkaline phosphatase may yield bone invasion. It is additionally essential to screen for AIDS, as NHL could be its initial clinical aspect. Liver and kidney functions ought to be explored [4].

Though, it's impossible to distinguish between HL and NHL solely on imaging, rest in sure it is indeed necessary for the staging. The routinely performed CT (head and neck, chest, abdomen and pelvis), is used to especially detect bone deterioration. MRI in the other hand is quite interesting, in matters of soft tissue infiltration; naming for example the tongue, infra-temporal fossa, the para-pharyngeal space and intracranial or intraspinal involvement. Typically, both NHL and HL typically present as homogeneous multiple nodes. Extra-nodal ones, more often than not showcase as homogeneous submucosa lesions, that ought to appear slightly enhanced postcontrast. Moreover, NHL tends to rebuild instead of destroying bone, though it can erode adjoining bone. Clinically aggressive lymphomas are characterized by pulverization of the mandible, maxillae, and sinus walls. [4], [16], [27], [28].

Positron emission tomography (PET) is solidly reliable and advanced than MRI and CT in identifying recurrent or residual lesions, along with recognizing and discriminating active residual tumors and posttreatment fibrosis [29].

The staging of this disease is eased by other procedures. A bone marrow biopsy shows bone involvement. All HIV patients should also benefice from lumbar puncture, along with any patients with specific histological subtypes, such as Burkitt and obviously in the case of neurological symptoms. The lumbar puncture is also performed to identify the possible destruction of the skull base [4], [30]. To note that the Ann Arbor staging system is the most used staging system [31].

Many studies were conducted to establish prognostic factors that are correlated with survival and/ or relapse rate, and that affect the overall treatment strategy; among those we find age, performance status, presence of extra-nodal lesions, hemoglobin level, cytogenetics, LDH level, immunophenotype, growth fraction, b2-microglobulin production, Ann Arbor clinical stage [4], [32], [33].

Treatment of extra-nodal NHL is based on surgical excision, often followed by chemotherapy, radiation therapy, or both. the standard treatment for the early stage diffuse large cell lymphoma consists in chemotherapy followed by radiotherapy. Oral cavity malignancy, though extensive, remain localized, hence the sufficiency of the treatment especially for the cheek lesion. Patients with advanced stage (bulky stage II, stage III and IV) have to be treated by combined chemotherapy. The most commonly used chemotherapy protocol is CHOP, it can be associated with a monoclonal antibody, rituximab with positive results in terms of survival. This modified protocol is named R-CHOP [1], [4], [7], [10], [34], [35].

\section{CONCLUSION}

In regards of Lymphomas, the main task of otorhinolaryngologists surgeons consists of a correct and early diagnosis, by obtaining a proper a biopsy specimen. Although the treatment usually performed by hematologists or oncologists, otorhinolaryngologists do participate regularly to detect any likable recurrence. 


\section{REFERENCES}

[1] Patini R, Coviello V, Riminucci M, Corsi A, Cicconetti A. Early-stage diffuse large B-cell lymphoma of the submental region: a case report and review of the literature. Oral Surgery.

[2] Epstein JB, Epstein JD, Le ND, Gorsky M. Characteristics of oral and paraoral malignant lymphoma: a population-based review of 361 cases. Oral Surg, Oral Med, Oral Patho, I Oral Radiol, Endod. 2001; 92: 519 25 .

[3] Van der Waal RI, Huijgens PC, Van der Valk P, Van der Waal I. Characteristics of 40 primary extranodal non-Hodgkin lymphomas of the oral cavity in perspective of the new WHO classification and the international prognostic index. Int J Oral Maxillofac Surg. 2005; 34 : 391-5.

[4] Zapater E, Bagan JV, Carbonell F, Basterra J. Malignant lymphoma of the head and neck. Oral Diseases. 2010; 16, 119-128.

[5] Fuessinger MA, Voss P, Metzger MC, Zegpi C, Semper-Hogg W. Numb Chin as Signal for Malignancy-Primary Intraosseous Diffuse Large B-Cell Lymphoma of the Mandible. Annals of Maxillofacial Surgery. 2018; 8(1).

[6] Kipps TJ. Chronic lymphocytic leukemia and related diseases. Williams Hematol. 2001; 6: 1163

[7] Sharma RK, Yadav VS, Kataria SP, Tewari S, Narula SC. Primary Non-Hodgkin Lymphoma of Palatal Gingiva. Clinical Advances in Periodontics. 2012; 2(1).

[8] Kolokotronis A, Konstantinou N, Christakis I, Papadimitriou P, Matiakis A, Zaraboukas T, Antoniades, D. Localized B-cell nonHodgkin's lymphoma of oral cavity and maxillofacial region: A clinical study. Oral Surg Oral Med, Oral Pathol Oral Radiol Endod. 2005; 99: 303-310.

[9] Farquhar D, Sobanko J, Williams K, Newman JG. A Vanishing Lymphoma in the Cheek. ORL. 2014; 76: 189-192.

[10] Malaguarnera M, Giordano M, Russo C, Puzzo L, Trainiti M, Consoli AS, Catania VE. Lymphoma of cheek: a case report. European Review for Medical and Pharmacological Sciences. 2012; 16(4 Suppl): 4-7.

[11] Shamloo N, Ghannadan A, Jafari M, Ahmadi S, Mortazavi H, Baharvand M. Head and Neck Lymphoma in an Iranian Population. Iranian Journal of Otorhinolaryngology. 2017; 3(5).

[12] Basirat M, Rabiei M, Bashardoust N. Incidence of Head and Neck Lymphoma in Guilan Province, Iran. Asian Pac J Cancer Prev. 2016; 17: 1-4.

[13] Etemad-Moghadam S, Tirgary F, Keshavarz S, Alaeddini M. Head and neck non-Hodgkin's lymphoma: a 20-year demographic study of 381 cases. Int. J. Oral Maxillofac. Surg. 2010; 39: 869-872.

[14] Jaffe ES, Harris NL, Stein H, Isaacson PG. Classification of lymphoid neoplasms: the microscope as a tool for disease discovery. Blood. 2008; 112: 4384-4399.

[15] Nathu RM, Mendenhall NP, Almasri NM, Lyunch JW. Non-Hodgkin's lymphoma of the head and neck: a 30-year experience at the University of Florida. Head Neck. 1999; 21: 247-254.

[16] Weber A, Rahemtullah A, Ferry JA. Hodgkin and non-Hodgkin lymphoma of the head and neck: clinical, pathologic, and imaging evaluation. Neuroimaging Clin N Am. 2003; 13: 371-392.

[17] Oluwasanmi AF, Wood SJ, Baldwin DL, Sipaul F. Malignancy in asymmetrical but otherwise normal palatine tonsils. Ear Nose Throat J. 2006; 85: 661-663.

[18] Anselmo AP, Cavalieri E, Cardarelli L, Gianfelici V, Osti F, Pescarmona E, et al. Hodgkin's disease of the nasopharynx: diagnostic and therapeutic approach with a review of the literature. Ann Hematol. 2002; 81: 514-516.

[19] Talmon Y, Gilbey P, Falah R, Samet A, Cohen H, Khoury J. Primary $\mathrm{B}$ cell lymphoma of the tongue. IMAJ. 2007; 9: 755-756.

[20] Kemp S, Gallagher G, Kabani S, Noonan V, O’Hara C. Oral nonHodgkin's lymphoma: review of the literature and World Health Organization classification with reference to 40 cases. Oral Surg Oral Med Oral Pathol Oral Radiol Endod. 2008; 105: 194-201.

[21] Lima I, Carneiro AS, Amorim CA, Santiago MB. Hodgkin lymphoma as a complication of primary Sjo"gren's syndrome. Mod Rheumatol. 2008; 18: 200-202.

[22] Patiar S, Ramsden JD, Freeland AP. B-cell lymphoma of the larynx in a patient with rheumatoid arthritis. J Laryngol Otol. 2005; 119: 646649.

[23] Cuadra-Garci'a I, Proulx GM, Wu CL, Wang CC, Pilch BZ, Harris NL, et al. Sinonasal lymphoma: a clinicopathologic analysis of 58 cases from the Massachusetts General Hospital. Am J Surg Pathol. 1999; 23: 1356-1369.

[24] Das DK, Francis IM, Sharma PN, Sathar SA, John B, George SS, et al. Hodgkin's lymphoma: Diagnostic difficulties in fine-needle aspiration cytology. Diagn Cytopathol. 2009; 37: 564-573.
[25] Harris NL, Jaffe ES, Stein H, Isaacson PG. A revised EuropeanAmerican classification of lymphoid neoplasm: a proposal from the International Lymphoma Study Group. Blood. 1994; 84: 1361-1392.

[26] Hwang JH, Kim DW, Kim KS, Lee SY. Mucosa-associated lymphoid tissue lymphoma of the accessory parotid gland presenting as a simple cheek mass A case report. Hwang et al. Medicine. 2019; 98: 36.

[27] Aiken A, Glastonbury C. Imaging Hodgkin and non-Hodgkin lymphoma in the head and neck. Radiol Clin North Am. 2008; 46: 363378.

[28] King AD, Lei KI, Richards PS, Ahuja AT. Non-Hodgkin's lymphoma of the nasopharynx: CT and MR imaging. Clin Radiol. 2003; 58: 621625 .

[29] Cremerius U, Fabry U, Neuerburg J, Zimny M, Osieka R, Buell U. Positron emission tomography with $18 \mathrm{~F}-\mathrm{FDG}$ to detect residual disease alter therapy for malignant lymphoma. Nucl Med Commun. 1998; 19: 1055- 1063 .

[30] Aisenberg AC. Coherent view of non-Hodgkin's lymphoma. J Clin Oncol. 1995; 13: 2656-2675.

[31] Moormeier JA, Williams SF, Golomb HM. The staging of nonHodgkin's lymphoma. Semin Oncol. 1990; 17: 43-50.

[32] Hermans J, Krol AD, van Groningen K, Kluin P, Kluin-Nelemans JC, Kramer $\mathrm{MH}$, et al. International prognostic index for aggressive nonHodgkin's lymphoma is valid for all malignancy grades. Blood. 1995; 86: 1460-1463.

[33] Johnston A, Salles G. Prognostic Systems for lymphoma. Hematol Oncol Clin North Am. 2008; 22: 839-861.

[34] Economopoulos T, Asprou N, Stathakls N, Fountzilas G, Pavlidis N, Papaspyrou S, et al. Primary extranodal non-Hodgkin's lymphoma of the head and neck. Oncology. 1992; 49(6): 484-488.

[35] Wang CC. Primary Malignant Lymphoma of the Oral Cavity and Paranasal Sinuses. Radiology. 1971; 100: 151-153. 